\title{
COMPRENDER LAS PRÁCTICAS EVALUATIVAS DEL PROFESOR DE MATEMÁTICAS PARA MEJORAR EL APRENDIZAJE DE LA FUNCIÓN
}

\author{
MATHEMATICS TEACHER EVALUATION TO IMPROVE \\ LEARNING OF LINEAR FUNCTIONS
}

Jhon Sebastian Gómez*

Elizabeth Hurtado Martínez**

\footnotetext{
* Magíster en Educación de la Universidad Surcolombiana. Docente de la Fundación Escuela Tecnológica de Neiva Jesús Oviedo Pérez. sebastian_gomez93@ hotmail.com 0000-0002-1564-3514

** Magíster en docencia de las matemáticas de la Universidad Pedagógica Nacional. Profesora tiempo completo de la Universidad de la Amazonia de Florencia, Caquetá.

e.hurtado@udla.edu.co 0000-0001-9374-4145
}

Cómo citar este artículo: Gómez, J. S. \& Hurtado, M. E. (2021). Comprender las prácticas evaluativas del profesor de matemáticas para mejorar el aprendizaje de la función. Revista PACA 11, pp. 107-138.
Resumen: El documento muestra los resultados del proyecto de investigación titulado "Comprender las prácticas evaluativas del profesor de matemáticas para mejorar el aprendizaje de la función lineal", definiendo como propósitos caracterizar las prácticas evaluativas que realiza el profesor de matemáticas asociadas al contenido matemático función lineal, identificar desde el modelo curricular Análisis Didáctico buenas prácticas evaluativas y proponer una estrategia metodológica para promover buenas prácticas de evaluación que aporten al mejoramiento en la comprensión de la función lineal en el aula de matemáticas.

Para caracterizar las prácticas evaluativas del profesor de matemáticas se identificó la tipología sugerida por Zambrano (2014), y para el diseño metodológico el Análisis Didáctico desde la perspectiva de Gómez (2015), que promoviera buenas prácticas evaluativas. Este referente aportó información relevante desde la didáctica de las matemáticas, las matemáticas mismas y el contexto para el diseño de las tareas matemáticas. Como producto final de esta investigación se tiene como resultado cinco tareas que buscan mejorar las prácticas evaluativas, utilizando el análisis didáctico. La propuesta de evaluación presentada motiva los intereses de los estudiantes, y busca generar escenarios de reflexión acerca de lo que están aprendiendo los estudiantes, cómo lo está aprendiendo y cuál es la responsabilidad del profesor en el diseño e 
implementación de estrategias de aula que permitan que los estudiantes se acerquen a los aprendizajes y capacidades.

Palabras clave: prácticas evaluativas, análisis didáctico y buenas prácticas.

\begin{abstract}
The document shows the results of the research project entitled "Understanding the evaluative practices of the mathematics teacher to improve the learning of the linear function", defining the purposes of characterizing the evaluative practices carried out by the mathematics teacher associated with the mathematical content linear function, identifying from the curricular model Didactic Analysis good evaluative practices and propose a methodological strategy to promote good evaluation practices that contribute to the improvement in the understanding of the linear function in the mathematics classroom.
\end{abstract}

To characterize the evaluative practices of the mathematics teacher, the typology suggested by Zambrano (2014) was identified and for the methodological design the Didactic Analysis from the perspective of Gómez (2015), which promoted good evaluative practices, this reference provided relevant information from the didactics of mathematics, mathematics itself and the context for the design of mathematical tasks.

108 Keywords: evaluative practices, didactic analysis, good practices.

\title{
Introducción
}

La pregunta que guió la investigación presentada fue: ¿Cómo promover buenas prácticas de evaluación que aporten al mejoramiento en el aprendizaje de la función lineal?. Para su desarrollo se planteó como propósito comprender las prácticas evaluativas del profesor de matemáticas con el fin de promover buenas prácticas de evaluación para el aprendizaje de la función lineal. En el marco de los intereses investigativos, las categorías conceptuales que se abordaron para su sustento teórico corresponden a los siguientes referentes: Evaluación: Popham (1990); Castillo y Cabrerizo (2003) y Zambrano (2014). Tipos de evaluación: Stenhouse (1984), Bordas y Cabrera (2001), Stiggins (2002), Eisner (2002), Litwin (2005), Freire (2002), Carr (2005) Moreno y Ortíz (2008), Flores y Gómez (2009), y Zambrano (2014). La evaluación de los aprendizajes en matemáticas: Chevellard (1991) y Rico (1997). Las buenas prácticas: González (2001), Epper y Bates (2004); Escudero, Gonzáles y Martínez (2009); De Pablos, Colás y González (2010) y Boza y Toscano (2011). El análisis didáctico como modelo curricular para promover buenas 
prácticas evaluativas: Rico (1997); Gómez (2006-2015); Cañadas, Gómez y Pinzón (2015) y Hurtado, Ochoa y Triviño (2017); y la función lineal como contenido matemático escolar: Lineamientos Curriculares para el área de Matemáticas (1998); Estándares Curriculares en Matemáticas (1997) y Derechos Básicos de Aprendizaje (2016).

En virtud a los elementos que fundamentan el referente teórico y a los intereses de la investigación, esta se sustentó en una perspectiva interpretativa y crítica de las ciencias sociales, asociada a los métodos cualitativos de la investigación social (Kemmis y Carr, 1986), los cuales reivindican la dimensión subjetiva de las prácticas investigativas en el aula de matemáticas.

Respecto a sus resultados, el proyecto permitió describir las prácticas evaluativas que ponen en funcionamiento los profesores en el aula de matemáticas, asociadas a la función lineal, identificadas desde el modelo curricular "Análisis Didáctico", buenas prácticas evaluativas asociadas al contenido a partir del reconocimiento de su diversidad de significados, formas de representación y los fenómenos que le dan sentido. Finalmente, el proyecto aportó una construcción metodológica para favorecer buenas prácticas evaluativas asociadas a la función lineal, desde el diseño de cinco tareas de aprendizaje, sustentadas desde el modelo curricular.

Los resultados de la investigación contribuyen a la educación matemática, aportando una propuesta metodológica innovadora que promueve mejoras en las prácticas evaluativas del profesor de matemáticas en el aula referidas al contenido función lineal, reconociendo desde las mismas matemáticas y el contexto, información útil y necesaria para su comprensión.

\section{Referentes teóricos}

Teniendo en cuenta los intereses de la investigación, las categorías conceptuales que la sustentan son: la evaluación, tipos de evaluación, la evaluación de los aprendizajes en matemáticas, las buenas prácticas, el análisis didáctico como modelo curricular para promover buenas prácticas evaluativas y la función lineal como contenido matemático escolar.

Respecto a la evaluación, Freire (2002) y Carr (2005) señalan que la evaluación simboliza el sendero que permite en los estudiantes el 
desarrollo de sus habilidades cognitivas y sociales, que los convertirán en seres autónomos y conscientes de sus responsabilidades sociales. Las prácticas evaluativas del profesor no deben sustentarse en la medición del conocimiento, sino en la promoción de competencias y habilidades para que los estudiantes se acerquen a las formas de pensar y resolver problemas que les permitan comprender el mundo en que habitan.

En coherencia a la perspectiva de Freire y Carr, Chevellar (1991), refiriéndose a la evaluación del aprendizaje de las matemáticas, señala que esta hace parte del funcionamiento didáctico en la relación de la tríada profesor, saber matemático y estudiante. La evaluación es parte del contrato didáctico, lo cual hace que no se convierta en una acción periférica del proceso didáctico, sino que hace parte de las reglas, las estrategias y los procesos de enseñanza, de aprendizaje y de comunicación.

En cuanto a las buenas prácticas, Escudero, Gonzáles, y Martínez (2009) señalan que la buena práctica no debe entenderse como algo objetivo, fijo o predeterminado y externo a la acción de los sujetos y sus contextos; requiere, más bien, ser construída a través de procesos juiciosos y propósitos morales situados en contextos, procesos que han de implicar reflexión e interpretación por parte de los actores y procesos que al mismo tiempo han de quedar expuestos a ser contrastados y validados en la acción debidamente fundada, no separados de ella. Los autores enfatizan que desde esta concepción, una buena práctica se entiende como un empeño reflexivo, interpretativo, dialogado y personal, también social y cultural.

Finalmente, Gómez (2006) identifica el Análisis Didáctico como una herramienta útil para el diseño de currículos locales (diseño de una clase de matemáticas) y se estructura alrededor de los organizadores del currículo. Estos últimos, según Rico (1997), se definen como elementos conceptuales y metodológicos que posibilitan al profesor la recolección, organización y selección de información acerca de los diferentes significados de los objetos matemáticos. El Análisis Didáctico como modelo curricular implica procesos de reflexión y análisis de las matemáticas, su enseñanza y su aprendizaje; este incorpora cuatro tipos de análisis que se sustentan desde los componentes curriculares: contenido (análisis de contenido), objetivos (análisis cognitivo), metodología (análisis de la instrucción) y evaluación (análisis de la actuación). Los tres primeros análisis se ocupan 
del diseño, mientras que el análisis de actuación se centra en la puesta en práctica, implementación y la posterior evaluación de los resultados obtenidos.

\section{Metodología}

La investigación se inscribe en un enfoque de investigación cualitativa, exploratoria y transformadora. Su carácter cualitativo se sustenta en que centra su interés en comprender las prácticas evaluativas que realiza el profesor de matemáticas; es de carácter exploratorio porque constituye un acercamiento a los escenarios del aula de clase de matemáticas para caracterizar las prácticas evaluativas que realiza el profesor, asociadas a la función lineal; y es de carácter transformador, porque su reconocimiento crítico busca promover buenas prácticas evaluativas en el aula de matemáticas a partir de un diseño metodológico desde un modelo curricular. Teniendo en cuenta los objetivos de la investigación, la estructura metodológica se sustentó en tres fases: caracterización, análisis y diseño. La Tabla 1 presenta las fases y su relación con los objetivos específicos, las actividades, categorías de análisis, fuentes e instrumentos de información.

Tabla 1

Estructura metodológica de la investigación.

\begin{tabular}{|c|c|c|c|c|c|}
\hline $\begin{array}{l}\text { Fases de la } \\
\text { investigación }\end{array}$ & $\begin{array}{l}\text { Objetivos } \\
\text { específicos }\end{array}$ & Actividades & $\begin{array}{l}\text { Categoría de } \\
\text { análisis }\end{array}$ & $\begin{array}{l}\text { Fuentes de } \\
\text { información }\end{array}$ & $\begin{array}{l}\text { Instrumentos } \\
\text { de recolección } \\
\text { de información }\end{array}$ \\
\hline $\begin{array}{l}\text { Fase de } \\
\text { caracterización }\end{array}$ & $\begin{array}{l}\text { Caracterizar } \\
\text { las prácticas } \\
\text { evaluativas } \\
\text { que realiza el } \\
\text { profesor de } \\
\text { matemáticas } \\
\text { asociadas al } \\
\text { contenido } \\
\text { matemático } \\
\text { función lineal. }\end{array}$ & $\begin{array}{l}\text { Descripción } \\
\text { del tipo de } \\
\text { prácticas } \\
\text { evaluativas } \\
\text { que realiza el } \\
\text { profesor de } \\
\text { matemáticas } \\
\text { cuando evalúa } \\
\text { los aprendizajes } \\
\text { asociados a la } \\
\text { función lineal. } \\
\end{array}$ & $\begin{array}{l}\text { Tipos de prácticas } \\
\text { evaluativas } \\
\text { según: } \\
\text { El momento } \\
\text { ¿Cuándo evaluar? } \\
\text { La finalidad } \\
\text { ¿Para qué } \\
\text { evaluar? } \\
\text { La extensión } \\
\text { ¿Qué } \\
\text { dimensiones } \\
\text { evaluar? } \\
\text { El origen de sus } \\
\text { agentes } \\
\text { ¿Quién evalúa? } \\
\text { Su normotipo } \\
\text { ¿Qué tipo de } \\
\text { referente? }\end{array}$ & $\begin{array}{l}\text { Observación de } \\
\text { clases } \\
\text { Revisión de } \\
\text { instrumentos } \\
\text { de evaluación } \\
\text { diseñados y } \\
\text { gestionados por el } \\
\text { profesor. } \\
\text { Entrevista a } \\
\text { profesores en } \\
\text { ejercicio. }\end{array}$ & $\begin{array}{l}\text { Rejilla de } \\
\text { observación. } \\
\text { Instrumentos de } \\
\text { evaluación. } \\
\text { Cuestionario de } \\
\text { entrevista. }\end{array}$ \\
\hline
\end{tabular}


COMPRENDER LAS PRÁCTICAS EVALUATIVAS DEL PROFESOR DE MATEMÁTICAS PARA MEJORAR EL APRENDIZAJE...

\begin{tabular}{|c|c|c|c|c|c|}
\hline $\begin{array}{l}\text { Fase de } \\
\text { Análisis }\end{array}$ & $\begin{array}{l}\text { Identificar } \\
\text { desde el análisis } \\
\text { didáctico } \\
\text { buenas prácticas } \\
\text { evaluativas para } \\
\text { el contenido } \\
\text { función lineal. }\end{array}$ & $\begin{array}{l}\text { Definición de } \\
\text { elementos } \\
\text { desde el } \\
\text { modelo } \\
\text { curricular } \\
\text { "análisis } \\
\text { didáctico" } \\
\text { que puedan } \\
\text { promover } \\
\text { buenas } \\
\text { prácticas } \\
\text { evaluativas } \\
\text { en el aula de } \\
\text { matemáticas. }\end{array}$ & $\begin{array}{l}\text { Desde el modelo } \\
\text { curricular: } \\
\text { ¿Cuándo evaluar? } \\
\text { ¿Para qué } \\
\text { evaluar? } \\
\text { ¿Qué } \\
\text { dimensiones } \\
\text { evaluar? } \\
\text { ¿Quién evalúa? } \\
\text { ¿Qué tipo de } \\
\text { referente? }\end{array}$ & $\begin{array}{l}\text { Organizadores } \\
\text { curriculares del } \\
\text { modelo curricular } \\
\text { análisis didáctico. }\end{array}$ & $\begin{array}{l}\text { Instrumentos } \\
\text { de análisis } \\
\text { del modelo } \\
\text { curricular }\end{array}$ \\
\hline Fase de diseño & $\begin{array}{l}\text { Proponer una } \\
\text { estrategia } \\
\text { metodológica } \\
\text { para promover } \\
\text { buenas prácticas } \\
\text { de evaluación } \\
\text { que aporten al } \\
\text { mejoramiento } \\
\text { en el aprendizaje } \\
\text { de la función } \\
\text { lineal. }\end{array}$ & $\begin{array}{l}\text { Diseño de } \\
\text { propuesta } \\
\text { metodológica } \\
\text { para promover } \\
\text { buenas } \\
\text { prácticas } \\
\text { evaluativas } \\
\text { en el aula de } \\
\text { matemáticas, } \\
\text { asociadas al } \\
\text { contenido } \\
\text { función lineal. }\end{array}$ & $\begin{array}{l}\text { ¿Qué significados } \\
\text { están asociados } \\
\text { al contenido } \\
\text { función lineal? } \\
\text { ¿Qué sistemas de } \\
\text { representación? } \\
\text { ¿Qué fenómenos } \\
\text { del contexto } \\
\text { social sustenta el } \\
\text { contenido? } \\
\text { ¿Qué objetivos } \\
\text { de aprendizaje, } \\
\text { capacidades y } \\
\text { competencias } \\
\text { matemáticas } \\
\text { promueve } \\
\text { el contenido } \\
\text { matemático? } \\
\text { ¿Qué dificultades } \\
\text { y errores se } \\
\text { pueden presentar } \\
\text { cuando se } \\
\text { aprende el } \\
\text { contenido } \\
\text { matemático? } \\
\text { ¿Qué tareas } \\
\text { matemáticas, } \\
\text { materiales } \\
\text { y recurso } \\
\text { didáctico se } \\
\text { pueden diseñar } \\
\text { para promover } \\
\text { buenas prácticas } \\
\text { evaluativas? } \\
\text { ¿Cómo } \\
\text { gestionar tareas } \\
\text { matemáticas y } \\
\text { como evaluar } \\
\text { los aprendizajes } \\
\text { logrados en los } \\
\text { estudiantes? }\end{array}$ & Modelo curricular & $\begin{array}{l}\text { Instrumentos } \\
\text { de análisis } \\
\text { del modelo } \\
\text { curricular. }\end{array}$ \\
\hline
\end{tabular}




\section{Análisis y tratamiento de datos}

En este apartado se presenta cómo se analizaron y se trataron los datos desarrollados en la investigación, por medio de la caracterización de las prácticas evaluativas asociadas a la función lineal que realizaron los profesores de matemáticas. Para ello, se asumieron como fuente de información: la observación de planeaciones y sesiones de clase, el desarrollo de entrevistas semiestructuradas y el análisis de los instrumentos de evaluación que diseñan y gestionan los profesores en el aula.

Las observaciones de las planeación y sesiones de clase

Las observaciones se realizaron teniendo en cuenta los diferentes momentos de la clase y las prácticas evaluativas que realizaron los profesores en cada uno de ellos; de manera específica: el momento uno, "preparación o planeación de la clase"; momento dos, "presentación o introducción del contenido en la clase"; momento tres, "desarrollo de la clase"; y momento cuatro, "cierre de la clase". La información se registró en la siguiente rejilla, teniendo como referentes los diferentes criterios tipológicos de evaluación sugeridos por Zambrano (2014). 


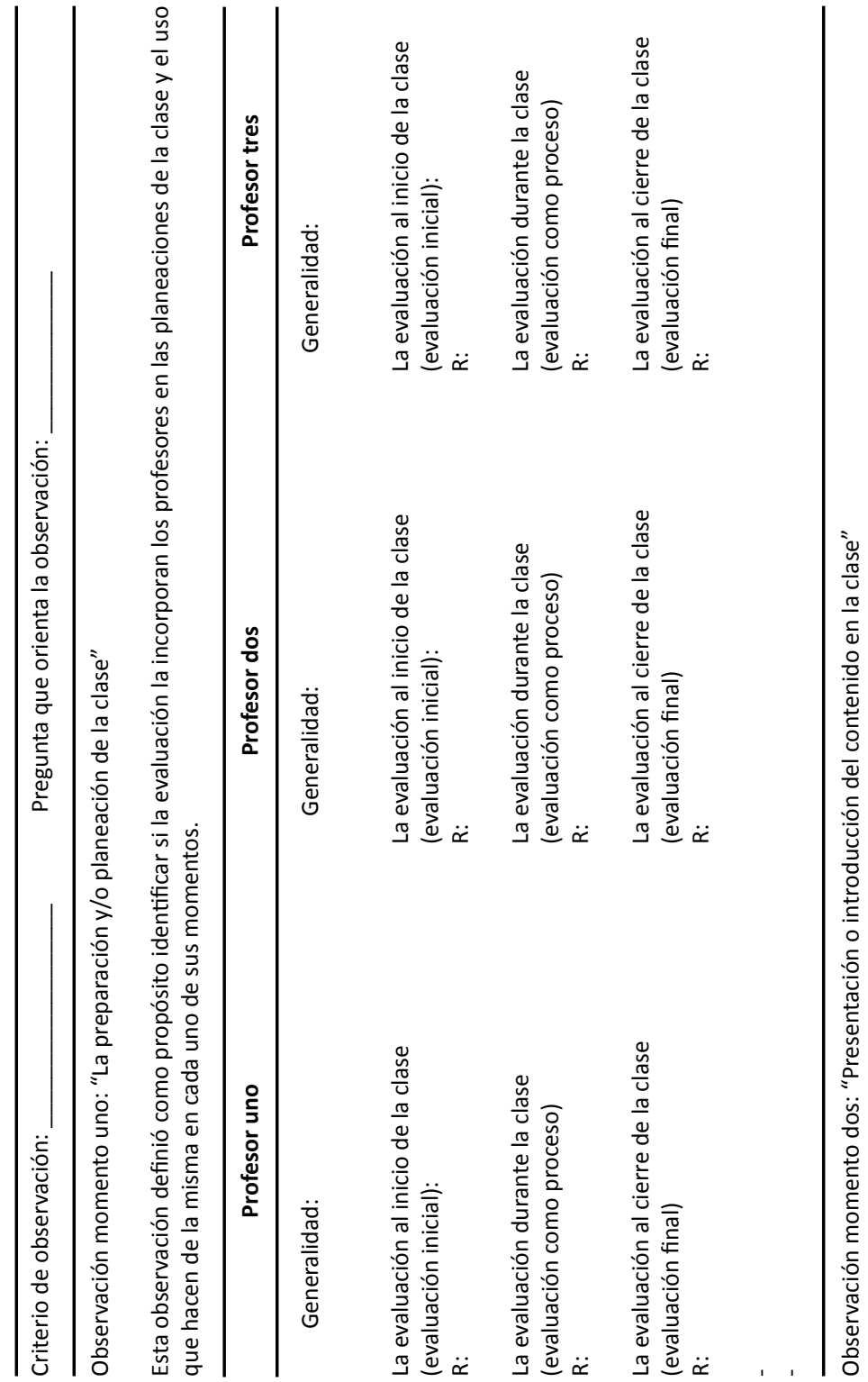

Esta observación se realizó con el interés de describir el uso que hacen los profesores de la evaluación en la presentación o introducción del contenido en el aula. 


\begin{tabular}{llc}
\hline Profesor uno & Profesor dos & Profesor tres \\
\hline Generalidad: & Generalidad: & Generalidad: \\
$\begin{array}{l}\text { A continuación se registra la } \\
\text { actuación del profesor en la que } \\
\text { hace uso de la evaluación. }\end{array}$ & $\begin{array}{l}\text { A continuación se registra la } \\
\text { actuación del profesor en la } \\
\text { que hace uso de la evaluación. }\end{array}$ & $\begin{array}{l}\text { A continuación se registra las } \\
\text { actuaciones del profesor en la } \\
\text { que hace uso de la evaluación. }\end{array}$ \\
\hline A1: & A1: & A1: \\
\hline
\end{tabular}

Observación momento tres: "Desarrollo de la clase"

Esta observación se realizó con el interés de describir el uso que hacen los profesores de la evaluación durante la enseñanza y el aprendizaje del contenido en el aula.

\begin{tabular}{lcc}
\hline \multicolumn{1}{c}{ Profesor uno } & Profesor dos & Profesor tres \\
\hline Generalidad: & & \\
& & \\
A continuación se presentan las actuaciones de los profesores observadas en las diferentes sesiones de clase, \\
que sustentan lo afirmado. & $\mathrm{A} 1:$ & $\mathrm{A} 1:$ \\
\hline $\mathrm{A} 1:$ & $\mathrm{A} 2:$ & $\mathrm{A} 2:$ \\
$\mathrm{A} 2:$ & & \\
\hline
\end{tabular}

Observación momento cuatro: "Cierre de la clase"

Esta observación definió como propósito identificar la forma como el profesor cierra el estudio del contenido en el aula y el papel de la evaluación en ese proceso.

\begin{tabular}{lcc}
\hline Profesor uno & Profesor dos & Profesor tres \\
\hline Generalidad: & \\
A continuación se presentan las actuaciones de los profesores observadas en las diferentes sesiones de clase, \\
que sustentan lo afirmado. \\
\hline $\mathrm{A} 1:$ & $\mathrm{A} 1:$ & $\mathrm{A} 1:$ \\
\hline
\end{tabular}

Fuente: Elaboración propia.

\section{Análisis de la información de las observaciones y planeación de las sesiones de clase}

Con la información obtenida a partir de observaciones de diferentes sesiones de aula se registra la caracterización de las prácticas evaluativas del profesor de matemáticas en la siguiente rejilla. 


\begin{tabular}{ll}
\hline Tipos de evaluación & $\begin{array}{c}\text { Caracterización de las prácticas evaluativas } \\
\text { del profesor de matemáticas }\end{array}$ \\
\hline Según el momento & Prácticas evaluativas en "la planeación de la clase" \\
& Prácticas evaluativas en el "desarrollo de la clase" \\
Respecto al criterio ¿cuándo evaluar?, se concluye que: & Prácticas evaluativas "en la planeación de la clase" \\
\hline Según la finalidad & Prácticas evaluativas en "el desarrollo de la clase" \\
\hline & Prácticas evaluativas en el "cierre de la clase" \\
\hline
\end{tabular}

Se concluye respecto al criterio ¿para qué evaluar?, que

Prácticas evaluativas "en la planeación de clase"

Según el origen de sus agentes Prácticas evaluativas "en el desarrollo de la clase"

Prácticas evaluativas "al cierre de la clase"

Respecto al criterio ¿quién evalúa? se concluye que

Prácticas evaluativas "en la planeación de clase"

Prácticas evaluativas en el "desarrollo de la clase"

Según el normotipo

Prácticas evaluativas en el "cierre de la clase"

Se concluye que frente a la cuestión ¿qué tipo de referentes usa el profesor al evaluar?,

la evaluación criterial

Fuente: Elaboración propia.

- Desarrollo de entrevistas semiestructuradas

Se realizaron entrevistas semiestructuradas a los profesores de matemáticas que permitieran identificar, desde sus discursos, las prácticas evaluativas que realizan asociadas a la función lineal. En la siguiente tabla se registró la información obtenida en las entrevistas realizadas, teniendo como referentes los diferentes tipos de evaluación sugeridos por Zambrano (2014). 


\begin{tabular}{lll}
\hline $\begin{array}{l}\text { Criterio: Según el momento } \\
\text { Pregunta orientadora: ¿Cuándo evaluar? }\end{array}$ & \\
\hline Profesor uno & Profesor dos & Profesor tres \\
\hline Al inicio: & Al inicio: & Al inicio: \\
Durante: & Durante: & Durante: \\
Al finalizar: & Al finalizar: & Al finalizar: \\
\hline
\end{tabular}

Se puede inferir que

Criterio: Según la finalidad

Pregunta orientadora: ¿Para qué evaluar?

\begin{tabular}{lll}
\hline Profesor uno & \multicolumn{1}{c}{ Profesor dos } & Profesor tres \\
\hline Para diagnosticar: & Para diagnosticar: & Para diagnosticar: \\
Para formar: & Para formar: & Para formar: \\
Para alcanzar desempeños: & Para alcanzar desempeños: & Para alcanzar desempeños: \\
\hline
\end{tabular}

Se puede inferir que

Criterio: Según la extensión

Pregunta orientadora: ¿Qué dimensiones evaluar?

\begin{tabular}{clc}
\hline Profesor uno & Profesor dos & Profesor tres \\
\hline Global: & Global: & Global: \\
Parcial: & Parcial: & Parcial: \\
\hline
\end{tabular}

Se logra identificar que

\begin{tabular}{|c|c|c|}
\hline \multicolumn{3}{|c|}{$\begin{array}{l}\text { Criterio: Según el origen de sus agentes } \\
\text { Pregunta orientadora: ¿Quién evalúa? }\end{array}$} \\
\hline Profesor uno & Profesor dos & Profesor tres \\
\hline Autoevaluación: & Autoevaluación: & Autoevaluación: \\
\hline Coevaluación: & Coevaluación: & Coevaluación: \\
\hline Heteroevaluación: & Heteroevaluación: & Heteroevaluación: \\
\hline
\end{tabular}

En cuanto 
Criterio: Según su normotipo

Pregunta orientadora: ¿Qué tipo de referente?

\begin{tabular}{lll}
\hline Profesor uno & Profesor dos & Profesor tres \\
\hline Normativo: & Normativo: & Normativo: \\
Criterial: & Criterial: & Criterial: \\
\hline
\end{tabular}

Se puede evidenciar que

Fuente: Elaboración propia.

\section{Análisis del desarrollo de entrevistas semiestructuradas}

La información obtenida en las entrevistas permite identificar características asociadas a las prácticas evaluativas de los profesores de matemáticas desde sus discursos. Se propuso la siguiente rejilla para su análisis.

\begin{tabular}{|c|c|}
\hline Tipos de evaluación & Caracterización de las prácticas evaluativas del profesor de matemáticas \\
\hline Según el momento & $\begin{array}{l}\text { Al inicio: } \\
\text { Durante: } \\
\text { Al finalizar: }\end{array}$ \\
\hline Según la finalidad & $\begin{array}{l}\text { Diagnóstica: } \\
\text { Formativa: } \\
\text { Sumativa: }\end{array}$ \\
\hline Según la extensión & $\begin{array}{l}\text { Global: } \\
\text { Parcial: }\end{array}$ \\
\hline Según el origen de sus agentes & $\begin{array}{l}\text { Autoevaluación: } \\
\text { Coevaluación: } \\
\text { Heteroevaluación: }\end{array}$ \\
\hline Según el normotipo & $\begin{array}{l}\text { Normativo: } \\
\text { Criterial: }\end{array}$ \\
\hline
\end{tabular}

Fuente: Elaboración propia. 


\section{Los instrumentos de evaluación que diseñan y gestionan los profesores en el aula}

En la siguiente tabla se registra la información obtenida de los instrumentos usados por los profesores en sus prácticas evaluativas. Se registró una tabla por cada instrumento analizado.

Criterio: Según la extensión

Pregunta orientadora: ¿Qué dimensiones evaluar?

Categorías de análisis

Descripción de prácticas matemáticas

(Capacidades matemáticas)

Capacidades desde el saber

C1: Identifica una función lineal en su representación tabular

C2: Identifica una función lineal en su representación gráfica

C3: Identifica una función lineal en su representación algebraica o simbólica

C4: Hace conversiones entre diferentes formas de representación de la función lineal

C5: Hace la gráfica de una función lineal

C6: Identifica la variable independiente y la variable dependiente en una función lineal

C7: Identifica el dominio, el codominio y el rango de una función lineal

C8: Explica por qué el codominio y el rango de una función lineal es el mismo

C9: Argumenta la relación entre una función lineal y la proporcionalidad entre dos magnitudes

C10: Halla el valor de la pendiente en una función lineal

C11: Explica el significado de una pendiente negativa en una función lineal

C12: Explica el crecimiento o decrecimiento en una función lineal

C13: Explica la incidencia en la gráfica de una función lineal cuando se suma o resta una cantidad cualquiera

C14: Extrapola valores en la gráfica de una función lineal 


\section{Capacidades desde el saber hacer}

C1: Identifica y expresa en lenguaje natural situaciones de la vida cotidiana que se puedan modelar con funciones lineales

C2: Grafica funciones utilizando recursos tecnológicos como computador (Geogebra, Derive, etc.) o su celular (aplicaciones como Malmath, Grapher Math Draw, Algeo, etc.)

C3: Modela situaciones de su vida cotidiana mediante una función lineal

C4: Explica el significado de la proporcionalidad en una situación cotidiana

C5: Resuelve problemas relativos a funciones lineales

C6: Crea problemas que se puedan modelar mediante funciones lineales

C7: Predice resultados en situaciones que se comporten como una función lineal

\section{Capacidades desde el saber ser}

C1: Aporto ideas al trabajo en equipo

C2: Apoyo a los compañeros que presentan alguna dificultad en algún tema

C3: Me apoyo en los compañeros para aclarar mis dudas

C4: Me esfuerzo por realizar todas las actividades propuestas

C5: Consulto por mi propia cuenta lo que se me dificulta y practico en casa

C6: Me esfuerzo por realizar mis trabajos con calidad

Fuente: Elaboración propia.

\section{Análisis de los instrumentos de evaluación que diseñan y gestionan los profesores en el aula.}

Criterio: Según la extensión

Pregunta orientadora: ¿Qué dimensiones evaluar? 


\section{Capacidades desde el saber}

C1: Identifica una función lineal en su representación tabular

C2: Identifica una función lineal en su representación gráfica

C3: Identifica una función lineal en su representación algebraica o simbólica

C4: Hace conversiones entre diferentes formas de representación de la función lineal

C5: Hace la gráfica de una función lineal

C6: Identifica la variable independiente y la variable dependiente en una función lineal

C7: Identifica el dominio, el codominio y el rango de una función lineal

C8: Explica por qué el codominio y el rango de una función lineal es el mismo

C9: Argumenta la relación entre una función lineal y la proporcionalidad entre dos magnitudes

C10: Halla el valor de la pendiente en una función lineal

C11: Explica el significado de una pendiente negativa en una función lineal

C12: Explica el crecimiento o decrecimiento en una función lineal

C13: Explica la incidencia en la gráfica de una función lineal cuando se suma o resta una cantidad cualquiera

C14: Extrapola valores en la gráfica de una función lineal

En los instrumentos de evaluación se evidencia

\section{Capacidades desde el saber hacer}

C1: Identifica y expresa en lenguaje natural situaciones de la vida cotidiana que se puedan modelar con funciones lineales

C2: Grafica funciones utilizando recursos tecnológicos como computador (Geogebra, Derive, etc.) o su celular (aplicaciones como Malmath, Grapher Math Draw, Algeo, etc.)

C3: Modela situaciones de su vida cotidiana mediante una función lineal 
COMPRENDER LAS PRÁCTICAS EVALUATIVAS DEL PROFESOR DE MATEMÁTICAS PARA MEJORAR EL APRENDIZAJE...

C4: Explica el significado de la proporcionalidad en una situación cotidiana

C5: Resuelve problemas relativos a funciones lineales

C6: Crea problemas que se puedan modelar mediante funciones lineales

C7: Predice resultados en situaciones que se comporten como una función lineal

En relación con las capacidades del saber hacer

Capacidades desde el saber ser

C1: Aporto ideas al trabajo en equipo

C2: Apoyo a los compañeros que presentan alguna dificultad en algún tema

C3: Me apoyo en los compañeros para aclarar mis dudas

C4: Me esfuerzo por realizar todas las actividades propuestas

C5: Consulto por mi propia cuenta lo que se me dificulta y practico en casa

C6: Me esfuerzo por realizar mis trabajos con calidad

Las capacidades desde el saber ser

Fuente: Elaboración propia.

\section{Resultados}

En este apartado se presentan los resultados logrados con el desarrollo de la investigación articulados a cada uno de los objetivos propuestos.

\section{Resultados asociados a la caracterización de las prácticas evaluativas que realiza el profesor de matemáticas asociadas al contenido matemático de la función lineal}

Para caracterizar las prácticas evaluativas que realiza el profesor de matemáticas en el aula, se acogió la tipología de evaluación propuesta por Zambrano (2014), quien realiza una adaptación a las aportaciones de Castillo y Cabrerizo (2010), para plantear una caracterización de 
la evaluación asociada a las siguientes cuestiones: ¿cuándo evaluar?, referida a los momentos de la evaluación; ¿para qué evaluar?, asociada a la finalidad de la evaluación; ¿qué dimensiones evaluar?, que definen si la evaluación es global o parcial; ¿quién evalúa?, que describe los actores que participan en la evaluación; y ¿qué tipo de referente sustenta la evaluación?, que son aquellas normas o criterios que sustentan la evaluación.

A partir de los elementos aportados desde el referente teórico, para caracterizar las prácticas evaluativas asociadas a la función lineal que realizaron profesores de matemáticas en ejercicio, se asumieron como fuentes de información: la revisión de las planeaciones, la observación a sesiones de clase, el desarrollo de entrevistas semiestructuradas y el análisis de los instrumentos de evaluación que diseñan y gestionan en el aula. A continuación se presentan los resultados obtenidos en cada asunto:

a) Caracterización desde la planeación de la clase de matemáticas: Se identificó que dentro de las componentes que sustentan el diseño: contenidos, objetivos, metodología y evaluación, esta última es la que menos considera el profesor; los escasos desarrollos que realizan están asociados a la aplicación de pruebas para conocer los desempeños de los estudiantes que permitan generar los reportes institucionales. Desde las cuestiones sugeridas por Zambrano (2014), se identificó que respecto a la pregunta ¿cuándo evaluar?, los profesores consideran evaluar al finalizar la clase, particularmente al cierre de la enseñanza del contenido, con el interés de conocer los niveles de alcance de logros de aprendizaje en los estudiantes; en cuanto a la cuestión ¿para qué evaluar?, los profesores evalúan para conocer desempeños de los estudiantes que permitan promover los reportes de notas. Esta perspectiva deja desprovisto el carácter formativo y permanente de la evaluación, reducida a evaluar, no para conocer la forma en que avanzan los estudiantes en el proceso formativo, sino para informar sobre los desempeños logrados, asignando un carácter instrumental al proceso de evaluar.

En cuanto a la pregunta ¿qué dimensiones se evalúan?, las planeaciones mostraron que los profesores centran el interés en la dimensión cognitiva sobre la dimensión afectiva; ninguna de las planeaciones registró interés por conocer cómo se siente el estudiante cuando aprende el contenido; el 
interés se fija en qué aprende del contenido, con una mirada fragmentada del mismo. En la misma perspectiva, al indagar sobre ¿quién evalúa?, se identifica únicamente la voz del profesor (heteroevaluación), y es desde su voz que se valoran las diferentes producciones esperadas en los estudiantes. Finalmente, respecto a la cuestión ¿qué tipo de referente sustenta la evaluación?, tal como se ha señalado, el único referente válido es el nivel de alcance de logros en los estudiantes.

b) Caracterización desde la gestión de la clase de matemáticas: Las prácticas de evaluación de los profesores se caracterizaron por: a) presentación expositiva del contenido; a los estudiantes, con ausencia de tareas que promuevan la comprensión del contenido, b) favorecimiento de la evaluación final como forma de validar si el estudiante resolvió de manera adecuada las actividades sugeridas, y no con intereses formativos que promovieran el aprendizaje de la función lineal a partir de las motivaciones y posibilidades de los estudiantes; c) los profesores se consideran los únicos agentes evaluadores dentro del proceso, ya que todas sus prácticas evaluativas se realizaron desde la heteroevaluación; y d) la evaluación desarrollada por los profesores asume como única norma válida el alcance de logros desde los desempeños de los estudiantes.

Por otra parte, los tres profesores desarrollaron prácticas de aula mediadas por talleres y actividades centradas en la resolución de ejercicios, de manera que los estudiantes pudieran aprender especialmente procesos algoritmizados de la función lineal, con ausencia de actividades que promovieran su comprensión. Desde esta perspectiva, los instrumentos y prácticas evaluativas se centraron en conocer los desempeños de los estudiantes desde la dimensión cognitiva, para generar los registros institucionales de calificaciones. Los profesores desconocen las formas de aprender de sus estudiantes, así como sus formas de negociar significados, validar los conceptos aprendidos, enfrentar y resolver las dificultades.

c) Caracterización desde los discursos de los profesores: Fue posible evidenciar que no hay coherencia entre los discursos de los profesores y lo que sucede en realidad en el aula. En las entrevistas los profesores reconocen el papel de la evaluación durante todo el proceso formativo, pero la reducen al cierre de la clase con intereses puramente cognitivos.

Desde las cuestiones sugeridas por Zambrano (2014), en los discursos de los profesores se identificó que en la cuestión ¿cuándo evaluar?, 
consideran importante evaluar al inicio de la clase para identificar los saberes previos de los estudiantes (evaluación inicial), durante el desarrollo de la clase (evaluación como proceso), para conocer la forma en que aprenden los estudiantes y al final de la clase para identificar las capacidades y logros alcanzados (evaluación final). En cuanto al ¿para qué evaluar?, los profesores expresaron la importancia de evaluar para conocer lo que saben los estudiantes antes de abordar el contenido (evaluación diagnóstica), para identificar la forma en que aprenden los estudiantes y acompañar la construcción de conocimiento matemáticos (evaluación formativa) y para determinar los desempeños alcanzados por los estudiantes (evaluación sumativa).

En lo que respecta a ¿qué dimensiones se evalúan?, los profesores consideran relevante evaluar desde la integralidad, es decir, desde el ser, el saber y el saber hacer; sin embargo, resaltan la importancia de la evaluación asociada al saber, sobre las otras dos. En lo referido a ¿quién evalúa?, los profesores consideran importante reconocer la voz de los estudiantes en la evaluación y, por supuesto, la suya. La coevaluación no está presente en sus relatos. En cuanto a ¿qué referente se evalúa?, tanto en la observación de clase como en las entrevistas se reconoce el alcance de los indicadores de logros concretos y prefijados por la institución como sustento para valorar de forma homogénea a los estudiantes y determinar el grado de dominio de la función lineal.

d) Caracterización desde el análisis de los instrumentos de evaluación: Este se realizó con el interés de identificar lo que evalúan los profesores de la función lineal. El análisis mostró el interés por conocer el dominio de los estudiantes asociados a la representación simbólica de la función lineal, seguida de la representación tabular y gráfica. Hay ausencia de conversiones entre los distintos sistemas de representación. Las actividades para evaluar están desprovistas de ambientes de aprendizaje enriquecidos con información del contexto; son actividades con uso de algoritmos sin la mediación de situaciones problema que impliquen el uso de la función lineal. Desde este referente se identifica el interés de evaluar con fines cognitivos asociados al saber, obviando el saber hacer y el ser. Los instrumentos diseñados permiten al profesor conocer los desempeños de los estudiantes; no se diseñan ni aplican instrumentos para conocer el avance en el aprendizaje desde la voz de los estudiantes; finalmente, solo se evalúa al cierre de la clase. 
Resultados asociados a la identificación de buenas prácticas evaluativas desde el análisis didáctico para el aprendizaje de la función lineal

El Análisis Didáctico incorpora conocimientos didácticos de las matemáticas para que los profesores fortalezcan el diseño de currículos locales (preparación de una clase de matemáticas). La siguiente tabla muestra la información que aporta el análisis didáctico para promover buenas prácticas desde la tipología de prácticas sugerida por Zambrano (2014).

\section{Tabla 2}

Buenas prácticas evaluativas desde el Análisis Didáctico.

\begin{tabular}{ccc}
\hline $\begin{array}{c}\text { Tipología de prácticas } \\
\text { evaluativas }\end{array}$ & $\begin{array}{c}\text { Prácticas evaluativas del profesor } \\
\text { desde el análisis didáctico }\end{array}$ & Elementos conceptuales \\
\hline
\end{tabular}

Según el momento ¿Cuándo evaluar?
Antes de cada sesión de clase, para identificar los conocimientos, capacidades y competencias matemáticas que activan los estudiantes al resolver tareas Inicial orientadas a este fin. Esto permite al profesor conocer las condiciones de los estudiantes frente a los objetivos de aprendizaje que se esperan alcanzar en la clase y tomar decisiones sobre la enseñanza del contenido.

Durante el desarrollo de las diferentes sesiones de la clase para lograr, a través de la gestión de diferentes tareas, conocer la forma como los estudiantes activan capacidades, fortalecen las competencias y conocimientos matemáticos, aportando información sobre el avance frente a los objetivos de aprendizaje definidos.

Al cierre de la clase, para identificar las capacidades que se activaron y las competencias matemáticas que se generaron en los estudiantes con Final la gestión de las tareas, así como los conocimientos matemáticos que se alcanzaron. Todo ello con el interés de mejorar tanto las prácticas de enseñar matemáticas de los profesores como los procesos de aprendizaje en los estudiantes.
Romero y Gómez (2015), frente a la pregunta ¿Cuándo evaluar desde el análisis didáctico?, señalan que en el contexto de la evaluación tradicional primero se enseña y luego se evalúa. El análisis didáctico se inscribe en el modelo de la evaluación formativa, la cual busca difuminar los límites entre la enseñanza y evaluación: tanto la enseñanza como la evaluación son componentes fundamentales del currículo.

Los autores enfatizan en que la evaluación se integra en la enseñanza y se concibe como un permanente proceso reflexivo, por parte tanto del profesor como de los estudiantes, apoyado en evidencias de diverso tipo, así las cosas, en el análisis didáctico se asume la evaluación imbricada en todos los momentos de la enseñanza. 
Diagnóstica

Según la

finalidad

¿Para qué evaluar?
Para conocer qué tanto saben los estudiantes frente al contenido, antes de iniciar el desarrollo de la clase. Se realiza a través de la gestión de tareas diagnósticas que le ofrezcan al profesor información sobre la condición inicial de los estudiantes frente a las expectativas de aprendizaje (objetivos de aprendizaje, capacidades y competencias matemáticas) que ha considerado para la clase y tomar decisiones respecto a la enseñanza del contenido (prácticas de enseñanza).

Este tipo de evaluación identifica, durante el desarrollo de la clase, el nivel de alcance de las expectativas de aprendizaje definidas para la sesión y acompaña a los estudiantes en la superación de errores y dificultades (previamente definidas por el profesor) que puedan incurrir en la búsqueda de garantizar el aprendizaje del contenido.

La evaluación sumativa está orientada a asignar una calificación al desempeño de los estudiantes, se transforma en una evaluación para describir desde la gestión de tareas matemáticas

Sumativa (pensada por el profesor con este interés) lo que el estudiante fue capaz de hacer a partir de las expectativas de aprendizaje (objetivos de aprendizaje, capacidades y competencias matemáticas) definidos.

El modelo del análisis didáctico, no solo reconoce la dimensión cognitiva (conocimientos y procedimientos) como importante en los procesos de formación, también reconoce la importancia de la dimensión afectiva. Esta dimensión aglutina un "extenso rango de sentimientos $y$ Global- humores (estados de ánimo) que son parcial generalmente considerados como algo diferente de la pura cognición, e incluye como componentes específicos de este dominio las actitudes, creencias y emociones" (McLeod y Adams, 1989, p. 245, citado por González y Gómez,

2014).
Romero y Gómez (2015), frente a la pregunta ¿Para qué evaluar desde el análisis didáctico?, señalan que en la evaluación tradicional, el propósito es determinar qué estudiantes tienen los conocimientos requeridos y cuáles no, y asignar calificaciones de acuerdo con ello. Este tipo de evaluación tiende a convertirse en un mecanismo de control, de selección, de comparación y de medición. Las instituciones se convierten en filtros sociales que han de clasificar a los sujetos en función de sus capacidades. En la perspectiva de la evaluación formativa, se evalúa para promover un aprendizaje matemático con comprensión en los estudiantes y para mejorar el proceso de enseñanza del profesor. Se pasa de una evaluación del aprendizaje a una evaluación para el aprendizaje. El fin último es ayudar a las personas a que crezcan y lleguen a sus máximas posibilidades.

Romero y Gómez (2015), frente a la pregunta ¿qué dimensiones evaluar desde el análisis didáctico?, señalan que en la evaluación tradicional se pone el énfasis en evaluar hechos específicos y destrezas aisladas, en forma de algoritmos y resolución de tareas rutinarias, a expensas de la comprensión, las conexiones y la resolución de problemas. extensión

¿Qué

dimensiones

evaluar? 
Se evalúa desde la integralidad del ser humano (estudiante que aprende), lo que la ubica en una dimensión global. Para cada dimensión, cognitiva y afectiva, se definen las capacidades que se espera lograr en los estudiantes con el aprendizaje del contenido matemático específico (función lineal), por lo que se evalúa desde la particularidad de ese contenido reconociendo la integralidad y complejidad de los estudiantes que lo aprenden.

Gómez y su equipo de colabores diseñaron una serie de instrumentos de evaluación que hacen participes a todos los autores en el proceso de evaluación. Desde esta perspectiva, importa tanto al profesor como al estudiante y a los demás estudiantes conocer el avance Autoevaluación

Según el origen de sus agentes ¿Quién evalúa? en el alcance de las expectativas de aprendizaje definidas para la clase. Los instrumentos diseñados incorporan la evaluación desde las dimensiones cognitiva y afectiva, así:

- El semáforo cognitivo: Evalúa la dimensión cognitiva desde la perspectiva de los estudiantes (nivel de activación de capacidades asociadas al saber).

Coevaluación • El matematógrafo: Evalúa la dimensión afectiva desde la perspectiva de los estudiantes (cómo se sintió al resolver las diferentes tareas matemáticas).

- El diario del profesor: Evalúa la forma en que los estudiantes van alcanzado las expectativas de aprendizaje definidas por el profesor, desde su propia perspectiva.
La evaluación formativa incorpora

la valoración de procesos de pensamiento, estrategias seguidas para la resolución de problemas, uso de materiales y recursos, habilidades de comunicación oral y escrita, actitudes y comportamientos, entre otros.

Por otra parte, la enseñanza del profesor también puede ser objeto de evaluación. En el caso del análisis didáctico, todo el análisis de actuación está encaminado a la evaluación de esa enseñanza, en términos de su planificación e implementación.

Romero y Gómez (2015), frente a la pregunta ¿quién evalúa desde el análisis didáctico?, señalan que la evaluación tradicional supone que, en la clase, solo el profesor puede evaluar adecuadamente el progreso de los estudiantes. Sin embargo, las experiencias avalan que la evaluación más efectiva de todas es la que uno realiza de su propio aprendizaje.

Una de las capacidades más valiosas que un estudiante puede lograr es la habilidad de mirar atrás y reflexionar sobre lo que ha hecho y sobre lo que todavía le queda por hacer. El hábito de autoevaluarse está relacionado con el desarrollo del potencial de aprendizaje continuo a lo largo de la vida. De ahí que la evaluación formativa incorpore a los estudiantes en el proceso y se responsabilice de que logren esa capacidad. Además, los estudiantes pueden desarrollar la capacidad de evaluar a sus compañeros (evaluación por pares o coevaluación) y al profesor. 
Según su normotipo ¿Desde qué referente se evalúa?
En el modelo del análisis didáctico, las expectativas de aprendizaje que define el profesor desde la planificación de clase se convierten en el referente desde el cual se evalúa; en este sentido, Normativo - criterial los objetivos de aprendizaje que se esperan alcanzar, las capacidades que se esperan activar y las competencias a que se espera contribuir con la gestión de tareas matemáticas, constituyen el referente desde el cual se evalúa.
Romero y Gómez (2015), frente a la pregunta ¿desde qué referente se evalúa desde el análisis didáctico?, señalan que la evaluación tradicional se apoya mayormente en los controles y exámenes para evaluar. Sin embargo, la formulación habitual de estas pruebas suele forzar a los estudiantes a aprender de forma superficial y los profesores se muestran cansados y aburridos cuando realizan las correcciones. La evaluación formativa supone que los estudiantes, desde las primeras etapas de su aprendizaje, necesitan tener una guía de cómo lo están haciendo, de cómo pueden avanzar y de cómo pueden superar los obstáculos que se lo impiden.

Los exámenes son útiles en un sistema mixto de evaluación, pero un examen formal, con calificaciones numéricas, no es suficiente para proporcionar a los estudiantes la guía que necesitan. Se deben incorporar tareas que visibilicen el pensamiento de los estudiantes, formulación de preguntas que propicien reflexión, fomento de la comunicación en el aula, trabajos escritos y diarios de los estudiantes, realimentación a través de comentarios a lo largo del proceso, registros de observación de la clase, y autoevaluación, entre otros. Los pilares de la evaluación son el criterio y la evidencia. Si hay que realizar juicios, los criterios deben ser explícitos y compartidos y deben mostrar las evidencias correspondientes. Los instrumentos de evaluación diseñados desde el análisis didáctico incorporan sistemáticamente la formulación de criterios de evaluación y la manera de compartirlos con los estudiantes.

Resultados asociados a la propuesta metodológica para promover buenas prácticas de evaluación que aporten al mejoramiento en el aprendizaje de la función lineal 
El diseño de la propuesta metodológica acogió los elementos conceptuales y procedimentales del modelo curricular Análisis Didáctico, lo que favoreció el diseño de tareas matemáticas que permitieran movilizar a los estudiantes a la solución de situaciones problema, en las que activen prácticas matemáticas $\mathrm{y}$, desde esas prácticas, activar capacidades y competencias orientadas al alcance de los objetivos de aprendizaje.

Se diseñaron cinco tareas matemáticas, cada una pensada para favorecer prácticas evaluativas asociadas a los diferentes momentos de enseñanza del contenido función lineal. Todas las tareas incorporaron el diseño de instrumentos que promovieron la autoevaluación, la coevaluación y la heteroevaluación, reconociendo todas las voces de los actores en el proceso de formación, especialmente construir conocimiento matemático a partir de las negociaciones con el otro.

Igualmente, cada una de las tareas incorpora actividades para situar la evaluación como un proceso permanente ligado a las prácticas de enseñanza: al inicio, durante y al cierre de la clase. Las tareas promueven la evaluación formativa, en la que no solo importa el saber, sino el saber hacer y el ser. La siguiente tabla consolida información respecto a las tareas diseñadas:

Tabla 3

Tarea matemáticas para promover buenas practicas evaluativas de la función lineal.

\begin{tabular}{|c|c|c|c|c|c|}
\hline $\begin{array}{l}\text { Momento de } \\
\text { la clase }\end{array}$ & $\begin{array}{l}\text { No. } \\
\text { tarea }\end{array}$ & $\begin{array}{c}\text { Nombre de la } \\
\text { tarea }\end{array}$ & $\begin{array}{c}\text { Tipo de } \\
\text { tarea }\end{array}$ & $\begin{array}{l}\text { Propósito } \\
\text { de la tarea }\end{array}$ & Contenidos \\
\hline \multirow[t]{2}{*}{ Inicio } & 1 & $\begin{array}{l}\text { Aprender } \\
\text { comprando }\end{array}$ & Diagnóstica & $\begin{array}{l}\text { Explorar los } \\
\text { conocimientos } \\
\text { previos que tienen } \\
\text { los estudiantes } \\
\text { respecto a las } \\
\text { características de } \\
\text { la función lineal } \\
\text { y su relación con } \\
\text { otros tipos de } \\
\text { funciones (afín y } \\
\text { constante). }\end{array}$ & $\begin{array}{l}\text { Uso del plano cartesiano, } \\
\text { uso de tablas, lenguaje } \\
\text { algebraico, operaciones } \\
\text { con números reales, } \\
\text { diferencia de la relación } \\
\text { de función, solución de } \\
\text { ecuaciones de primer } \\
\text { grado, notación funcional y } \\
\text { hallar el valor numérico de } \\
\text { expresiones algebraicas. }\end{array}$ \\
\hline & 2 & $\begin{array}{l}\text { Aprendiendo } \\
\text { con los autos }\end{array}$ & $\begin{array}{l}\text { De } \\
\text { aprendizaje: } \\
\text { presentación } \\
\text { del contenido } \\
\text { matemático } \\
\text { función lineal. }\end{array}$ & $\begin{array}{l}\text { Lograr que los } \\
\text { estudiantes } \\
\text { construyan } \\
\text { significados de } \\
\text { conceptos básicos } \\
\text { de la función }\end{array}$ & $\begin{array}{l}\text { Variable dependiente } \\
\text { e independiente, } \\
\text { dominio, rango, relación } \\
\text { de proporcionalidad, } \\
\text { pendiente de una función } \\
\text { lineal con su respectivo }\end{array}$ \\
\hline
\end{tabular}


Desarrollo

4

COVID-19

De aprendizaje: Construcción de modelos usando la función lineal.

Cierre

De evaluación: Conocer
De aprendizaje: Sistemas de representación de la función lineal. lineal: identifique variable dependiente $e$ independiente, dominio, rango, relación de proporcionalidad, pendiente de una función lineal con su respectivo significado y su incidencia en la gráfica.

Lograr que los estudiantes exploren y comprendan diversas formas de representar la función lineal en situaciones de cambio y variación.

Lograr que los estudiantes construyan modelos usando la función lineal para comprender situaciones cotidianas de cambio y variación.

Identificar los conocimientos 5 Transporte y aprendizajes logrados. significado y su incidencia en la gráfica, así como en su interpretación en términos de la situación dada.

Formas de representación de la función, algebraica, tabular y gráfica.
Modelación de funciones lineales, gráficas de funciones lineales por medio de recursos tecnológicos, la proporcionalidad en situaciones cotidianas, creación y predicción de problemas en situaciones que se comporten como una función lineal.

Representar gráficamente en el plano cartesiano, identificar los elementos de una función, resolver y proponer problemas de la vida diaria. matemáticas que los estudiantes adquirieron durante las sesiones anteriores. 
Tabla 4

Objetivos de aprendizaje, capacidades y competencias matemáticas asociadas a la función lineal.

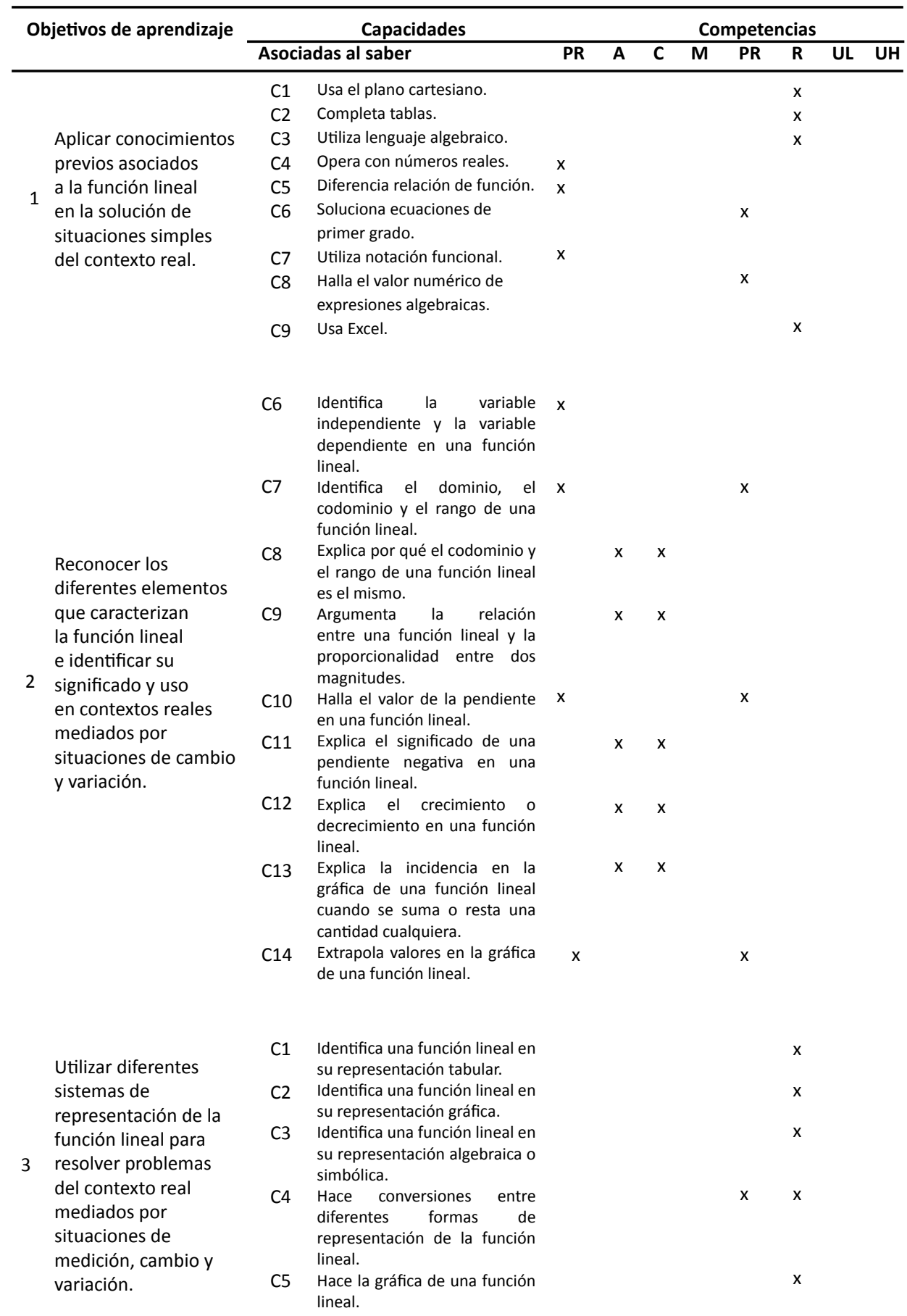


Formular y desarrollar modelos que describan situaciones

4 real entre magnitude físicas, que incorporen el uso de la función lineal para su solución.

Desarrollar habilidades para aprender la función lineal con la

5 participación de los demás compañeros, el profesor y por sí mismo.

\section{Asociadas al saber}

C1

Identifica y expresa en lenguaje natural situaciones de la vida cotidiana que se puedan modelar con funciones lineales.

C2 Grafica funciones utilizando recursos tecnológicos como el Excel.

C3 Modela situaciones de su vida cotidiana mediante una función lineal.

C4 Explica el significado de la proporcionalidad en una situación cotidiana.

C5 Resuelve problemas relativos a funciones lineales.

C6 Crea problemas que se puedan modelar mediante funciones lineales.

C7 Predice resultados en situaciones que se comporten como una función lineal.

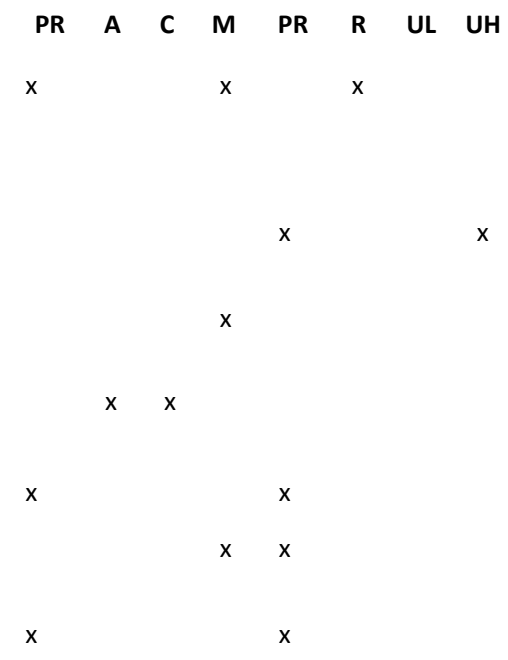

Las competencias corresponden a las definidas en el proyecto PISA: Pensar y razonar (PR), Argumentar (A), Comunicar (C), Modelar (M), Plantear y resolver problemas (PR), Representar (R), Utilizar el lenguaje simbólico, formal y técnico y las operaciones (UL), Usar herramientas y recursos (UH).

Para este documento se presenta el diseño de la tarea matemática cuatro, la cual acoge el objetivo de aprendizaje 4 registrado en la tabla anterior:

Actividades para evaluar al inicio de la clase: conocimientos previos. 


\section{Descripción de la tarea:}

El COVID-19 ha provocado la muerte de personas en todo el mundo; para prevenir su contagio existen los elementos de protección personal, como el tapabocas, guantes y gel antibacterial. Teniendo en cuenta que varios padres de familia del salón han tenido que suspender sus actividades laborales por el confinamiento decretado por el gobierno nacional, uno de los padres consideró vender tapabocas como posibilidad de ingresos para su casa. A continuación, se registran los tipos, costos de los tapabocas y quiénes los compran:

\begin{tabular}{clcl}
\hline No. & \multicolumn{1}{c}{ Tipo de tapabocas } & Valor por unidad & \multicolumn{1}{c}{ Usuarios } \\
\hline T1 & Tapabocas desechable con filtro & $\$ 996$ & Ciudadanos en general \\
T2 & Tapabocas tres capas, lavable & $\$ 2000$ & Personal de servicio doméstico \\
T3 & Tapabocas filtro N95 micropartículas & $\$ 5000$ & Operarios y jardineros \\
T4 & Tapabocas N95 antivirus con filtro & $\$ 7900$ & Taxistas \\
& & & \\
\hline
\end{tabular}

El papá de Santiago logró identificar el siguiente comportamiento de la venta de tapabocas durante cada una de las cuatro semanas de los meses de abril y mayo en su negocio, teniendo en cuenta el tipo de clientes que los compran:

- La primera semana inició con un comportamiento en ventas en cada tipo de tapabocas como se registra en la siguiente tabla:

Comportamiento ventas $\mathrm{S} 1$, mes de abril según tipo de tapabocas

\begin{tabular}{cc}
\hline Tipo de tapabocas & Cantidad de ventas \\
\hline T1 & 200 \\
T2 & 28 \\
T3 & 50 \\
T4 & 80 \\
\hline
\end{tabular}

- Cada semana la venta de los tapabocas T1 aumentó en un $15 \%$, lo tipo $\mathrm{T} 2$ en u $2 \%$, los tipos $\mathrm{T3}$ en un $5 \%$ y los tipos T4 en un $10 \%$.

Con la información registrada realiza las siguientes actividades:

A1: Registra en tablas la información que se deriva de la venta de cada uno de los tipos de tapabocas durante las diferentes semanas de los meses de análisis. Registra las conclusiones que obtienes.

A2: Ubica la información que obtienes de las tablas asociadas al comportamiento de ventas de los diferentes tipos de tapabocas en diferentes planos cartesianos. Registra las conclusiones que obtienes. 
A3: Teniendo en cuenta que ya conoces los diferentes significados de la pendiente de la recta, describe para este caso qué sentido le asignas en la venta de tapabocas.

A4: Con la información identificada, construye la ecuación lineal que muestra el comportamiento de las ventas en los meses de abril y mayo de cada tipo de tapabocas.

Con las actuaciones de los estudiantes para encontrar las soluciones a las diferentes preguntas planteadas en el contexto de la tarea, se espera que los estudiantes identifiquen cómo, a través de unos datos contextualizados, se pueden formular y desarrollar modelos que describan situaciones involucrando la función lineal.

\section{Actividades para evaluar el desarrollo de la clase}

De acuerdo con el problema anterior y con la tabla que se presenta, registra la información correspondiente y responde las diferentes cuestiones.

\begin{tabular}{|c|c|c|c|c|c|c|c|c|c|c|c|}
\hline $\begin{array}{l}\text { Tipo de } \\
\text { tapabocas }\end{array}$ & Semanas & $\begin{array}{l}\text { Cant. } \\
\text { Ventas }\end{array}$ & $\begin{array}{c}\text { Tipo de } \\
\text { tapabocas }\end{array}$ & Semanas & $\begin{array}{l}\text { Cant. } \\
\text { Ventas }\end{array}$ & $\begin{array}{c}\text { Tipo de } \\
\text { tapabocas }\end{array}$ & Semanas & $\begin{array}{l}\text { Cant. } \\
\text { Ventas }\end{array}$ & $\begin{array}{c}\text { Tipo de } \\
\text { tapabocas }\end{array}$ & Semanas & $\begin{array}{r}\text { Cant. } \\
\text { Ventas }\end{array}$ \\
\hline \multirow{3}{*}{$\begin{array}{c}\text { T1 } \\
\text { Mes abril }\end{array}$} & $\mathrm{S} 1$ & & \multirow{3}{*}{$\begin{array}{c}\text { T2 } \\
\text { Mes abril }\end{array}$} & $\mathrm{S} 1$ & & \multirow{3}{*}{$\begin{array}{c}\text { T3 } \\
\text { Mes abril }\end{array}$} & S1 & & \multirow{3}{*}{$\begin{array}{c}\text { T3 } \\
\text { Mes abril }\end{array}$} & S1 & \\
\hline & $\mathrm{S} 2$ & & & S2 & & & S2 & & & $\mathrm{S} 2$ & \\
\hline & S3 & & & S3 & & & S3 & & & S3 & \\
\hline & S4 & & & S4 & & & S4 & & \multirow{3}{*}{ T3 } & S4 & \\
\hline \multirow{3}{*}{$\begin{array}{c}\text { T1 } \\
\text { Mes mayo }\end{array}$} & S1 & & & S1 & & \multirow{2}{*}{ T3 } & S1 & & & S1 & \\
\hline & $\mathrm{S} 2$ & & $\mathrm{~T} 2$ & $\mathrm{~S} 2$ & & & $\mathrm{~S} 2$ & & & $\mathrm{~S} 2$ & \\
\hline & S3 & & Mes mayo & S3 & & Mes mayo & S3 & & Ivies mayo & S3 & \\
\hline & S4 & & & S4 & & & S4 & & & S4 & \\
\hline
\end{tabular}

A1: ¿Cómo es el comportamiento de las ventas en cada tipo de tapabocas?

A2: ¿Qué diferencias estableces en la cantidad de ventas de cada tipo de tapabocas? A3: ¿A qué le atribuyes las diferencias?

A4: ¿Qué analizas del tipo de tapabocas y los roles de las personas que los compran?

A5: ¿Qué tipo de tapabocas usas y por qué?

A6: ¿Consideras importante usar el tapabocas y por qué? ¿En dónde debes usarlo? A7: Ubica la información que obtienes de la tabla en un solo plano cartesiano y registra conclusiones.

Con las actuaciones de los estudiantes para encontrar soluciones a las diferentes preguntas planteadas en el contexto de la tarea, se espera que identifiquen cómo, a través de unos datos contextualizados, se pueden graficar ecuaciones en el plano cartesiano, y formular y desarrollar modelos que describan situaciones involucrando la función lineal. 


\section{Actividades para evaluar el cierre de la clase:}

Teniendo en cuenta la información suministrada respecto a las dinámicas de ventas de los diferentes tapabocas:

A1: Diseña para el papá de Santiago un análisis financiero que le permita conocer cuál ha sido la cantidad de dinero que ha ganado teniendo en cuenta:

El valor de compra de los tapabocas, así: T1: \$650, T2: \$1240, T3: \$3150, T4: $\$ 6480$; igualmente, que a cada tapabocas le debe adicionar $\$ 100$, de gastos operativos (mensajería, gastos de envíos, etc.).

Con la información suministrada, usando la representación que consideres, muestra los gastos generados al papá de Santiago en la compra de los diferentes tapabocas (no olvides considerar los gastos operativos).

A2: Realiza el análisis de cada gasto generado.

A3: Construye la ecuación lineal que muestre las dinámicas de gastos.

A4: Realiza un análisis entre las ventas y los gastos; para ello utiliza gráficas y tablas, que le permitan conocer al papá de Santiago las ganancias por cada tipo de tapabocas y las ganancias totales.

Con las actuaciones de los estudiantes para encontrar las soluciones a las diferentes preguntas planteadas en el contexto de la tarea, se espera que identifiquen cómo formular y desarrollar modelos que describan situaciones involucrando la función lineal.

\section{Conclusiones}

La investigación permitió reconocer el papel de la evaluación en los procesos de enseñanza del contenido matemático en cada uno de los momentos de la clase; igualmente permitió identificar desde el Análisis Didáctico información útil para favorecer buenas prácticas evaluativas en el aula de matemáticas, prácticas que aprovechan la riqueza del contenido, del contexto y de los estudiantes que aprenden. En la misma perspectiva se contribuyó en mostrar las aportaciones de la evaluación formativa en el favorecimiento de la comprensión del contenido, al conocer no solo lo que aprenden los estudiantes en términos del saber sino, especialmente, cómo se sienten mientras aprenden, desde el ser y saber hacer. Otro elemento a destacar es el alto componente participativo en la evaluación, no solo del profesor, quien tradicionalmente es quien evalúa, sino especialmente de los estudiantes. 


\section{Referencias Bibliográficas}

Bordas, M., y Cabrera, F. (2001). Estrategias de Evaluación de Aprendizaje. Revista Española de Pedagogía, (1) 1, 25-48.

Cañadas, M., Gómez, P., y Pinzón, A. (2015). Analisis de contenido. Obtenido de http://funes.uniandes.edu.co/11904/1/Canadas2018Analisis.pdf

Carr, D. (2005). El Sentido de la Educación. Una introducción a la filosofia y a la teoría de la educación y de la enseñanza. (1ed) Barcelona: Editorial Graró.

Castillo, S., y Cabrerizo, J. (2010). Evaluación educativa de aprendizajes y competencias. (1ed). Madrid: Uned - Pearson.

Chevellard, I. (1991). La transposición didáctica. Del saber sabio al saber enseñado. (1 ed). Buenos Aires: AIQUE.

De Pablos, J., Colás, P., y González, T. (2010). Un análisis comparativo entre diferentes políticas educativas au. Revista de Educación. (1)1. 23-51.

Eisner, E. (2002). La escuela que todos necesitamos. (1ed). Barcelona: Paidós.

Escudero, J., Ginzález, M., y Martínez, B. (2009). El fracaso escolar como exclusión educativa: Comprensión, políticas y prácticas.1(1) Revista Iberoamericana de Educación, 41-64.

Flores, Á., y Gómez, A. (agosto de 2009). Aprender Matemática, haciendo Matemática: la evaluación en el aula. Educación Matemática, 29(2), 117142.

Freire, P. (2002). Pedagogía de la Autonomía. Saberes necesarios para la práctica educativa. (1ed). México: Siglo XXI Editores.

Gómez, P. (2015). Análisis didáctico de la práctica de la formación de profesores de matemáticas. Tesis Doctoral Bogota. Obtenido de http://funes.uniandes. edu.co/11903/1/Gomez2018Analisis.pdf

González, F. A. (2001). Autorregulación del aprendizaje: una difícil tarea. Obtenido de: IberPsicologi: http://www.fedap.es/ IberPsicologia/iberpsi6-1/ gonzalez/gonzalez.htm

González, M. J., y Gómez, P. (2014). Análisis didáctico en la práctica de la formación permanente de profesores de matemáticas de secundaria. Módulo 3. Análisis Cognitivo. (1ed) Bogotá: Centro de Investigación y Formación en Educación (CIFE). Universidad de los Andes.

Hurtado, E., Ochoa, M., y Triviño, A. (2017). Diseño, gestión y evaluación de un programa de formación de profesores de matemáticas y física: hacia la consolidación de una comunidad de práctica. (1ed). Colombia: Universidad de la Amazonía.

Kemmis, S., y Carr, W. (1986). Teoría crítica de la enserianza. La investigaciónacción en la formación del profesorado. (1ed). Universidad de Barcelona.

Litwin, E. (2005). La evaluación: campo de controversias y paradojas o un nuevo lugar para la buena enseñanza. En A. Camilloni, La evaluación de los aprendizajes en el debate didáctico contemporáneo 1(1) (11-34). 
MEN. Ministerio de Educación Nacional. (1997). Estándares Curriculares en matemáticas. Bogotá. https:/www.mineducacion.gov.co/1621/ articles-116042_archivo_pdf2.pdf

MEN. (1998). Lineamientos Curriculares para el área de matemáticas. Bogotá. http://roychacon/lineamientos/agradecimientos.asp (mineducacion.gov.co)

MEN. (2016). Derechos Basicos del aprendizaje. Bogotá. aprende. colombiaaprende.edu.co/sites/default/files/naspublic/DBA_Matemáticas. pdf

Moreno, I., y Ortiz, J. (2008). Docentes de Educación Básica y sus concepciones acerca de la evaluación en matemática. Revista Iberoamericana de Evaluación Educativa, 1(1), 141-154.

Popham, W.J. (1990). Problemas y técnicas de evaluación educativa. (1ed). Anaya. Madrid.

Rico, L. (1997). Consideraciones sobre el currículo de matemáticas para educación secundaria. Granada, España: Universidad de Granada. Departamento Didática de la Matemática. Obtenido de http://funes.uniandes. edu.co/521/2/RicoL97-2528.PDF

Romero, I., y Gómez, P. (2015). Apuntes sobre análisis de actuación. Módulo 5 de MAD 3. Bogotá: Universidad de los Andes. Obtenido de http://funes. uniandes.edu.co/6886/

138 Morata.

Stiggins, R. (2002). Assessment Crisis: The absence of assessmentment for Learning. Phi Delta Kappan, 1(1). 758-765.

Zambrano, A. (2014). Prácticas evaluativas para la mejora de la calidad del aprendizaje: Un estudio contextualizado en La Unión-Chile. Tesis doctoral Bellaterra, Chile: Universidad Autónoma de Barcelona. Biblioteca digital. https:/www.tdx.cat/bitstream/handle/10803/284147/azd1de1. pdf? sequence $=1$ 